\title{
Effect of Risk Factors on Local and Systemic Control in Stage 1 Endometrial Cancer
}

\author{
(1) Binnur DÖNMEZ YILMAZ, (1) Özge KANDEMIR GÜRSEL \\ Department of Radiation Oncology, Prof. Dr. Cemil Taşcıoğlu City Hospital, İstanbul-Turkey
}

\begin{abstract}
OBJECTIVE
This study aims to evaluate the treatment outcomes according to the risk groups of patients with Stage 1 endometrial cancer and to investigate the effects of the risks associated with the disease in the control of the disease.

\section{METHODS}

Three hundred and ninety patients who were treated with the indication of endometrial cancer in our clinic were evaluated with the approval of the ethics committee. The data of 114 patients who were evaluated retrospectively between 2014-2018.

\section{RESULTS}

In patients with a median age of 58 (35-89) years, histological distribution of pathologies revealed the presence of cases of the 107 endometrioid adenocarcinoma (93\%), two serous (1.7\%), two clear cells $(1.7 \%)$, one mucinous $(0.8 \%)$ carcinomas and two $(1.7 \%)$ carcinosarcoma. High risk was detected in 14 (12.28\%), high to intermediate risk in nine (7\%), intermediate risk in 35 (30.7\%), and low-risk in $56 \mathrm{p}$ (49\%) patients. Recurrence developed in five (4\%) patients whose survival was found to be statistically significantly lower than those without recurrence (logrank: $41.74 \mathrm{p}=0.0001)$. Five -year survival rate was $95 \%$ and the mean $( \pm \mathrm{SD})$ survival time was $78.46 \pm 1.27$ months.
\end{abstract}

\section{CONCLUSION}

In the treatment of Stage 1 endometrial cancer, the survival rates achieved in our disease-recurrence group was statistically significantly lower than those who did not develop recurrences (logrank: 41.74 $\mathrm{p}=0.0001$ ).

Keywords: Brachytherapy; endometrial cancer; radiotherapy; risk factors.

Copyright $\odot$ 2020, Turkish Society for Radiation Oncology

\section{Introduction}

Endometrial cancer is the fourth highest form of cancer found in females in our country.[1] In endometrial cancer, which is most commonly seen in women over 50 years of age, obesity, metabolic syndrome and unopposed estrogen have been shown as etiologic factors. Clinicopathologically, endometrioid adenocarcinomas are seen in $80 \%$ of type 1 carcinomas, while type 2 carcinomas have a poor prognosis, and consist of serous, mucinous, clear cell and undifferentiated carcinoma types.[2] Features, such as tumor type, grade, myometrial invasion, lymphovascular invasion (LVSI) and age, are the prognostic significance for recurrence.[3-5]

European Society for Radiotherapy and Oncology (ESTRO) evaluates risk factors in low, intermediate, 
low-intermediate and high risk groups due to their effects on disease-free survival and survival (Table 1).[6] This grouping allowed patient-specific treatment planning, and the achievement of better disease-free survival and survival with the least possible side effects is targeted.

Ongoing molecular studies give an idea about the prognosis, although they have not yet taken their place in the treatment of endometrial cancer. Hypermethylation of PTEN, KRAS, CTNNB1 and PIK3CA and MLH1 promoters have been associated with type 1 cancers. The presence of P53 mutation in severe carcinomas often suggests a poor prognosis, and the L1 adhesion protein L1CAM is a determinant for distant metastasis. In patients with a POLE mutation, a very good prognosis is reported and adjuvant therapy is not recommended.[7]

In $75 \%$ of the cases, the tumor is inside the uterus at the time of diagnosis, and it spreads directly to the cervix or pelvic and then to the paraaortic lymph nodes. While pelvic lymph node metastasis is seen at a rate of $10 \%$ in the early stage, this rate increases by up to $20 \%$ as myometrial invasion increases and tumor differentiates.[8]

When radiotherapy is not applied to the patient in the high risk group in the postoperative period, it can be seen that locoregional recurrence occurred in the vaginal stump in $75 \%$ of the cases. PORTEC1 and GOG99 studies, which showed the effect of radiotherapy in local control, pioneered further studies.[5,9]

\section{Table 1 Factors that determine the risk groups as pro-} posed by ESTRO

\begin{tabular}{lc} 
Risk group & Definition \\
\hline Low risk (LR) & 1A Grade 1-2 LVI (-) \\
Intermediate risk (IR) & 1B Grade 1-2 LVI (-) \\
High-Intermediate risk (HIR) & 1A Grade 3 LVI ( \pm ) \\
High risk (HR) & 1B Grade 3 LVI ( \pm ) \\
\hline
\end{tabular}

This study aims to evaluate the treatment outcomes according to the risk groups of patients with Stage 1 endometrial cancer and to examine the effects of the risks associated with the disease in the control of the disease.

\section{Materials and Methods}

Three hundred and ninety patients, who were treated with the indication of endometrial cancer in our clinic, were evaluated with the approval of the ethics committee. Data of 114 patients with Stage 1 endometrial cancer patients admitted after surgery between 2014-2018 were retrospectively evaluated. Pathological reports, treatment results and radiological images of patients were obtained from automation system of our hospital.

In this study, statistical analyzes were performed with the NCSS (Number Cruncher Statistical System) 2007 Statistical Software (Utah, USA) package program. Overall (OS) and disease-free (DFS) survival rates of the study group were calculated and the descriptive statistical methods (mean, standard deviation, frequency and percentage distributions) were used in the evaluation of the data. The results were evaluated at the significance level of $\mathrm{p}<0.05$.

\section{Results}

The characteristics of the patients with a median age of 58 (35-89) years which may be related to the disease are shown in Table 2. Diabetes was the most common comorbidity in 27 patients (23\%) followed by hypertension in 19 patients (16\%) with or without diabetes. Eighty percent of patients consisted of postmenopausal women entered the menopause at a median age of $50(30-58)$ years. The first complaints were bleeding in $53 \%$, discharge in $21 \%$ and pain in $20 \%$ of the patients. Besides this, $1 \%$ of the patients were diagnosed by chance. The general characteristics of the patients are summarized in Table 2. Based on histologi-

Table 2 General characteristics of the patients

\begin{tabular}{lcccc} 
& Mean \pm SD & Median & Minimum & Maximum \\
\hline Age at menarche & $13.59 \pm 1.48$ & 13 & 11 & 17 \\
Age at first delivery & $22 \pm 5.04$ & 3 & 15 & 38 \\
Parity & $3.29 \pm 1.49$ & 3.5 & 1 & 7 \\
Tumor diameter & $3.49 \pm 1.81$ & 0.7 & 0.2 & 9.5 \\
Depth of invasion & $0.87 \pm 0.73$ & 2 & 0.1 & 4.5 \\
Thickness of myometrium & $2.02 \pm 0.74$ & 20 & 0.5 & 6 \\
Dissected lymph nodes $(\mathrm{n})$ & $25.12 \pm 18.67$ & 34.44 & 1 & 84
\end{tabular}


cal distribution of the cases, endometrioid adenocarcinoma $(\mathrm{n}=107(93.84 \%)$, serous $(\mathrm{n}=2: 1.77 \%)$, clear cell $(\mathrm{n}=2: 1.77 \%)$, mucinous type $(\mathrm{n}=1: 0.88 \%)$ carcinomas, and carcinosarcoma $((\mathrm{n}=2: 1.77)$ were identified in respective number of patients.

As shown in Table 3, the patients were treated with total abdominal hysterectomy and bilateral salpingo-opherectomy (TAH-BSO). Combined with pelvic $(n=31: 50 \%)$ and pelvic and paraaortic $(n=31: 50 \%)$ lymph node dissections in the respective number of patients. Distribution of patients into risk groups according to risk factors is shown in Table 4.

Ir-192 after loading brachytherapy system was used in patients undergoing brachytherapy. One of two radiotherapy sessions per week, and 550-700 cGy were applied on the vaginal surface for 3-5 times.

In patients undergoing pelvic radiotherapy, organs at risk were better protected with the use of intensive modulated radiotherapy (IMRT) technique and 46-50 Gy was applied. In the high-risk group, after external radiotherapy, $600 \mathrm{cGy}$ was applied once a week, as were three applications on the vaginal surface.

Patients in the low-risk group were solely followed up, if there were no additional risk factors, and patients in the high-risk group were treated with external and intracavitary brachytherapy (Table 5).

According to grade, there was no statistically significant difference between the survival times of the groups (LogRank: 0.26 p=0.877) (Table 6).

\begin{tabular}{lcc} 
Table $3 \quad$ Surgical interventions applied & \\
& $\mathbf{n}$ & $\%$ \\
\hline Type of surgery & & \\
TAH-BSO & 48 & 42.11 \\
TAH BSO+LND or Full Stage & 51 & 44.74 \\
TAH BSO LND Omentectomy & 11 & 8.77 \\
TAH BSO Omentectomy & 5 & 4.39 \\
\hline
\end{tabular}

TAH-BSO+LND: Total abdominal hysterectomy and bilateral salpingo-opherectomy and lymph node dissection

\begin{tabular}{lcc} 
Table 4 & $\begin{array}{l}\text { Distribution of patients into risk groups accord- } \\
\text { ing to risk factors }\end{array}$ \\
\hline HR & n & $\%$ \\
HIR & 14 & 12.28 \\
IR & 9 & 7.89 \\
LR & 35 & 30.70 \\
\hline
\end{tabular}

HR: High risk; HIR: High-Intermediate risk; IR: Intermediate risk; LR: Low risk

\begin{tabular}{lcc}
\hline Table 5 & Radiotherapy techniques applied & \\
& $\mathbf{n}$ & $\%$ \\
\hline Type of Radiotherapy & & \\
Observation & 36 & 31.58 \\
Brachytherapy & 55 & 48.25 \\
External+brachytherapy & 14 & 12.28 \\
External radiotherapy & 9 & 7.89 \\
\hline
\end{tabular}

In the group with myometrial invasion less than $50 \%, 2-, 3-$, and 5 year-survival rates were $0.978,0.978$, and 0.978 , respectively, while overall mean survival time was $75.95 \pm 1.11$ months. In the group with myometrial invasion more than $50 \%, 2-, 3-$, and 5 yearsurvival rates were $0.971,0.900$, and 0.900 , respectively, while overall mean survival time was $62.67 \pm 2.38$ months, without any statistically significant difference between the survival times of the groups (LogRank: $2.19 \mathrm{p}=0.138$ ). Overall disease free survival and survival of all patients at 2-,3-,5 years is shown in Table 7 .

The number of LVSI data is not sufficient to make the same assessment. Because three of five patients who developed recurrence did not have invasion and in two patients presence of invasion (if any) was not reported.

None of the patients died in the $<60$ age group. In the $\geq 60$ age group 2-, 3-, and 5- year survival rates were $0.949,0.919$, and 0.877 , with an overall mean survival time was $59.68 \pm 2.35$ months. Therefore, patients over 60 are considered to be in the high risk group (Fig. 1).

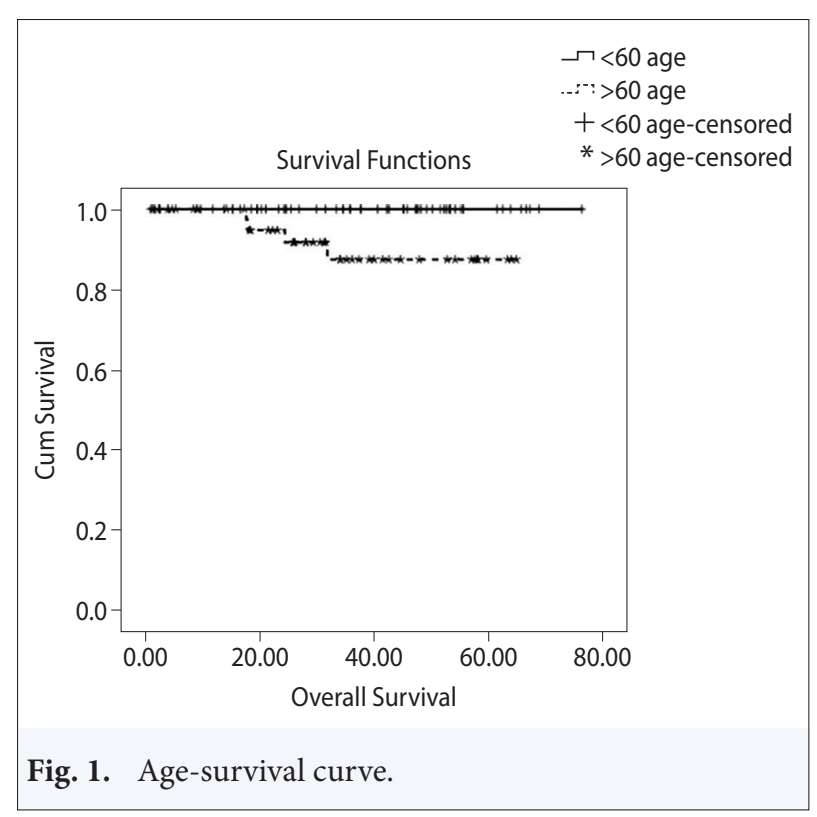


Table 6 Correlation between tumor grade and survival times

\begin{tabular}{lccr} 
& Grade 1 & Grade 2 & Grade 3 \\
\hline 1 year & & & 0.956 \\
2 year & & 0.956 & 0.917 \\
3 year & 0.966 & 0.956 & 0.917 \\
5 year & 0.966 & $64.81 \pm 1.52$ & $59.75 \pm 2.44$ \\
Median \pm SE Survival time & $74.29 \pm 1.75$ & $61.84-67.78$ & $54.96-64.54$ \\
$95 \% \mathrm{Cl}$ & $70.85-77.73$ & & \\
& Log rank:0.26 $\mathrm{p}=0.877$ & \\
\hline
\end{tabular}

Table 7 Overall disease-free survival

\begin{tabular}{lcc} 
& Disease-free survival & Survival \\
\hline 1 year & 0.989 & 0.989 \\
2 year & 0.978 & 0.978 \\
3 year & 0.966 & 0.966 \\
5 year & 0.950 & 0.950 \\
Median \pm SE Survival time & $73.47 \pm 1.28$ & $78.46 \pm 1.27$ \\
$95 \% \mathrm{Cl}$ & $70.97-75.97$ & $70.96-75.96$ \\
\hline
\end{tabular}

Survival rates in patients with recurrence $(+)$ were statistically significantly higher than those without recurrence (LogRank: 41.74 p=0.0001) (Fig. 2).

\section{Discussion}

Although guidelines are published for the surgical and postoperative treatment approaches of early-stage endometrial cancer, assessments of risks continue. In the

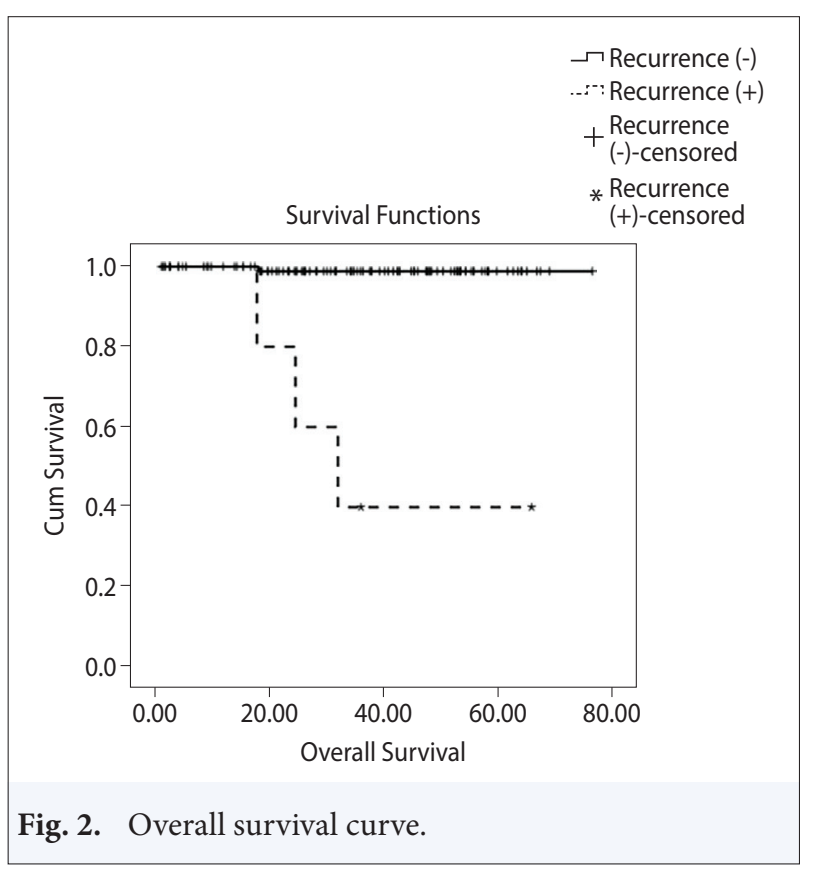

light of published treatment guidelines, the surgical methods were performed in patients with Stage 1 endometrial cancer in our clinic, and then, subsequent oncological approaches and the effects of risks on treatment outcomes were evaluated retrospectively.

In cancer surgery, lymphadenectomy is an integral part of staging. However, its role in early endometrial cancer is uncertain. Discussions about indications, anatomical scope, and therapeutic value of lymphadenectomy are still ongoing. In Panici and ASTEC studies, it has been reported that pelvic lymphadenectomy does not provide disease-free survival and survival advantage in early stage endometrial cancer, whereas in the metanalysis of nine studies by Kim et al. it was reported that extended lymph dissection did not provide benefit, especially in low-risk patients, and offered survival advantage in intermediate and high-risk patients. $[8,10,11]$

Decision should be made at the time of diagnosis according to histological type, the evaluation of myometrium thickness by ultrasonography, tumor grade, magnetic resonance imaging findings, and findings detected during surgery. Colombo et al. indicated that the number of lymph nodes to be removed in patients undergoing lymphadenectomy affects prognosis as much as risk factors.[12] In the metanalysis by Kim et al., the presence of 10-11 dissected lymph nodes has been shown to have an effect on survival in intermediate and high-risk patients. [11]

Pelvic and para-aortic lymph node dissections were performed in $53.5 \%$ of our patients. It was found that this application was not specific to a certain risk group due to variability in approaches between clinics. Two of the four patients with pelvic recurrence underwent complete lymphatic dissection, and two underwent TAH-BSO only.

In the GOG 99 study, the presence of lymphovascular invasion, age 60 and older, myometrial invasion and higher tumor grade were reported to be the factors that worsen the prognosis, and in PORTEC 1 and 2 trials, it 
was concluded that the presence of grade 3 disease, and lymphovascular invasion in the tumor was the most important risk factor for recurrence.

Of the five patients with recurrent disease, three patients had no lymphovascular invasion and the presence of lymphovascular invasion (if any) was not reported in two patients. Any statistically significant difference was not observed between the survival times in groups categorized according to tumor grades (Log Rank: 0.26 $\mathrm{p}=0.877$ ). Any statistically significant difference was not observed between groups with $<50 \%$ and $\geq 50 \%$ myometrial invasion as for 5-year survival times (LogRank: $2.19 \mathrm{p}=0.198$ ). However, all patients with disease recurrence were over 60 years of age and showed compliance with the literature concerning risks.

The success of external radiotherapy in local control after TAH-BSO in Stage 1 endometrial cancer was evaluated in the twenty-centered PORTEC1 study. Success in local control evaluated based on the results of 15 years of follow-up was also associated with increasing side effects over the years. However, this approach did not contribute to survival.[9] GOG 99, MRC ASTEC and MCIC CTG EN 5 studies also supported this finding. [5,13,14]

In the study in which the observation was randomized with brachytherapy in the low-risk group, brachytherapy contributed to local control, although it did not show a statistically significant difference. Brachytherapy could not contribute to survival in this group.[15] Accordingly, in our clinic, it was decided to follow up 36 of 56 patients in the low-risk group, while 20 patients underwent intracavitary brachytherapy for reasons, such as age, tumor diameter and lower uterine segment placement. One of the low-risk patients who were only monitored developed vaginal cuff and in another patient pelvic recurrence was detected. Molecular markers have not been studied in these patients. Curative radiotherapy was applied to the patient who developed vaginal cuff recurrence, and the patient who developed pelvic recurrence received only systemic treatment without surgery.

External pelvic radiotherapy was found to improve survival in patients with deep myometrial invasion in 21,249 endometrial cancer patients evaluated in the SEER database.

Besides, the addition of intracavitary radiotherapy to external radiotherapy in the intermediate -risk patient group has been reported to contribute to survival.[16] Radiotherapy in the high-risk group has been shown to provide survival advantage, regardless of the method applied.[17] Although intracavitary brachytherapy was often used in combination with ex- ternal radiotherapy, it was observed that two patients who developed pelvic recurrence had received pelvic radiotherapy. Molecular markers were not studied in these patients.

In the PORTEC -2 randomized study, intermediate- high-risk patients with Grade 1-2 tumors without deep lymphovascular invasion but with deep myometrial invasion were randomized to pelvic radiotherapy or vaginal brachytherapy. Despite similar local diseasecontrol rates, 5-year overall, and disease-free survival rates were better in the brachytherapy arm.[18] Based on this information, 55 patients (48\%) underwent vaginal intracavitary brachytherapy and one patient in this group developed pelvic recurrence.

Clinical outcomes were evaluated retrospectively in a single-center study. Although the number of patients was sufficient, the data obtained were not conclusive since the approach was not homogeneous in the risk groups. In addition, since molecular studies were not performed on our patients, our patient group could not be evaluated in the light of this information. The evaluation of patients with molecular studies in the subsequent pathology reports is expected to guide different studies.

\section{Conclusion}

In Stage 1 endometrial cancer, the survival rates achieved in our relapse-developing group were statistically significantly lower than patients who did not develop recurrence. Due to the effect of development of recurrence on survival, it is believed that, besides the risk factors, other molecular markers supporting the treatment decisions will help to create more homogeneous risk groups.

Acknowledgement: We would like to thank to Rana Konyalıoğlu for statistical evaluations.

Peer-review: Externally peer-reviewed.

Conflict of Interest: The authors declare that no conflict of interest.

Ethics Committee Approval: Ethics committee approval was received for this study as a retrospective study.

Financial Support: The authors received no financial supports.

Authorship contributions: Concept - B.D.Y.; Design B.D.Y.; Materials - B.D.Y.,Ö.K.G.; Data collection and/or processing - Ö.K.G.; Data analysis and/or interpretation B.D.Y.,Ö.K.G.; Literature search - B.D.Y.; Writing - B.D.Y.; Critical review - Ö.K.G. 


\section{References}

1. Cancer Statistics Turkey 2015. Available at: https:// hsgm.saglik.gov.tr/depo/birimler/kanser-db/istatistik/ Trkiye_Kanser_statistikleri_2015.pdf. Accessed Aug 20, 2020.

2. American College of Obstetricians and Gynecologists. ACOG practice bulletin, clinical management guidelines for obstetrician-gynecologists, number 65, August 2005: management of endometrial cancer. Obstet Gynecol 2005;106(2):413-25.

3. Kong A, Johnson N, Kitchener HC, Lawrie TA. Adjuvant radiotherapy for stage I endometrial cancer. Cochrane Database Syst Rev 2012;(3):CD003916.

4. Creasman WT, Morrow CP, Bundy BN, Homesley HD, Graham JE, Heller PB. Surgical pathologic spread patterns of endometrial cancer. A Gynecologic Oncology Group Study. Cancer 1987;60(8 Suppl):2035-41.

5. Keys HM, Roberts JA, Brunetto VL, Zaino RJ, Spirtos NM, Bloss JD, et al. A phase III trial of surgery with or without adjunctive external pelvic radiation therapy in intermediate risk endometrial adenocarcinoma: a Gynecologic Oncology Group study. Gynecol Oncol 2004;92(3):744-51.

6. Colombo N, Creutzberg C, Amant F, Bosse T, González-Martín A, Ledermann J, et al. ESMO-ESGO-ESTRO Consensus Conference on Endometrial Cancer: Diagnosis, Treatment and Follow-up. Int J Gynecol Cancer 2016;26(1):2-30.

7. Cancer Genome Atlas Research Network, Kandoth C, Schultz N, Cherniack AD, Akbani R, Liu Y, et al. Integrated genomic characterization of endometrial carcinoma. Nature 2013;497(7447):67-73.

8. ASTEC study group, Kitchener $H$, Swart AM, Qian Q, Amos C, Parmar MK. Efficacy of systematic pelvic lymphadenectomy in endometrial cancer (MRC ASTEC trial): a randomised study. Lancet 2009;373(9658):125-36.

9. Creutzberg CL, Nout RA, Lybeert ML, Wárlám-Rodenhuis CC, Jobsen JJ, Mens JW, et al. Fifteen-year radiotherapy outcomes of the randomized PORTEC-1 trial for endometrial carcinoma. Int J Radiat Oncol Biol Phys 2011;81(4):631-8.

10. Benedetti Panici P, Basile S, Maneschi F, Alberto Lissoni A, Signorelli M, Scambia Get al. Systematic pelvic lymphadenectomy vs. no lymphadenectomy in ear- ly-stage endometrial carcinoma: randomized clinical trial. J Natl Cancer Inst 2008;100(23):1707-16.

11. Kim HS, Suh DH, Kim MK, Chung HH, Park NH, Song YS. Systematic lymphadenectomy for survival in patients with endometrial cancer: a meta-analysis. Jpn J Clin Oncol 2012;42(5):405-12.

12. Lutman CV, Havrilesky LJ, Cragun JM, Secord AA, Calingaert B, Berchuck A, et al. Pelvic lymph node count is an important prognostic variable for FIGO stage I and II endometrial carcinoma with high-risk histology. Gynecol Oncol 2006;102(1):92-7.

13. Bosse T, Peters EE, Creutzberg CL, Jürgenliemk-Schulz IM, Jobsen JJ, Mens JW, et al. Substantial lymph-vascular space invasion (LVSI) is a significant risk factor for recurrence in endometrial cancer--A pooled analysis of PORTEC 1 and 2 trials. Eur J Cancer 2015;51(13):1742-50.

14. Greven K, Winter K, Underhill K, Fontenesci J, Cooper J, Burke T. Final analysis of RTOG 9708: adjuvant postoperative irradiation combined with cisplatin/paclitaxel chemotherapy following surgery for patients with high-risk endometrial cancer. Gynecol Oncol 2006;103(1):155-9.

15. Sorbe B, Nordström B, Mäenpää J, Kuhelj J, Kuhelj D, Okkan S, et al. Intravaginal brachytherapy in FIGO stage I low-risk endometrial cancer: a controlled randomized study. Int J Gynecol Cancer 2009;19(5):8738.

16. Homesley HD, Filiaci V, Gibbons SK, Long HJ, Cella D, Spirtos NM, et al. A randomized phase III trial in advanced endometrial carcinoma of surgery and volume directed radiation followed by cisplatin and doxorubicin with or without paclitaxel: A Gynecologic Oncology Group study. Gynecol Oncol 2009;112(3):543-52.

17. Harkenrider MM, Adams W, Block AM, Kliethermes S, Small W Jr, Grover S. Improved overall survival with adjuvant radiotherapy for high-intermediate and high risk Stage I endometrial cancer. Radiother Oncol 2017;122(3):452-57.

18. Nout RA, Smit VT, Putter H, Jürgenliemk-Schulz IM, Jobsen JJ, Lutgens LC, et al. Vaginal brachytherapy versus pelvic external beam radiotherapy for patients with endometrial cancer of high-intermediate risk (PORTEC-2): an open-label, non-inferiority, randomised trial. Lancet 2010;375(9717):816-23. 